\title{
Torsional Motion of the Chromophore Catechol following the Absorption of Ultraviolet Light
}

\author{
J. D. Young, ${ }^{1}$ M. Staniforth, ${ }^{1}$ M. J. Paterson, ${ }^{2}$ and V. G. Stavros ${ }^{1, *}$ \\ ${ }^{1}$ Department of Chemistry, University of Warwick, Gibbet Hill Road, Coventry CV4 7AL, United Kingdom \\ ${ }^{2}$ Institute of Chemical Sciences, Heriot-Watt University, Edinburgh EH14 4AS, United Kingdom
}

(Received 4 February 2015; published 8 June 2015)

\begin{abstract}
The ability to probe energy flow in molecules, following the absorption of ultraviolet light, is crucial to unraveling photophysical phenomena. Here we excite a coherent superposition of vibrational states in the first excited electronic state $\left(S_{1}\right)$ in catechol, resulting in a vibrational wave packet. The observed quantum beats, assigned to superpositions of the low-frequency, and strongly mixed, $\mathrm{O}-\mathrm{H}$ torsional mode $\tau_{2}$, elegantly demonstrate how changes in geometry upon photoionization from the $S_{1}$ state to the ground state of the cation $\left(D_{0}\right)$ enables one to probe energy flow at the very early stages of photoexcitation in this biological chromophore.
\end{abstract}

DOI: 10.1103/PhysRevLett.114.233001

PACS numbers: 33.15.-e

There has been concerted effort in recent years to characterize the excited state dynamics, following ultraviolet (UV) excitation, of several heteroatom-containing aromatic species, including phenols, pyrroles, azoles, and indoles (see, for example, Refs. [1-4]). These species are ubiquitous in nature, being key chromophores in a multitude of biological molecules [4-7]. By studying isolated chromophores in the absence of external perturbations (e.g., solvent, $p \mathrm{H}$, and temperature effects), the so-called "bottomup" approach, one can garner insight into the intrinsic properties of these chromophores and how these properties are carried through to larger biological molecules [4,8-12].

In this Letter, we apply such a bottom-up approach in the study of the excited state dynamics of catechol (1,2dihydroxybenzene), the structure of which is shown in Fig. 1(a). The catechol subunit is incorporated in a number of biologically relevant species such as eumelanin [5,13,14], the brown pigment copolymer found in human skin, which serves as a front line defense to UV radiation exposure. It was revealed in previous investigations [15-17] that, following photoexcitation from the ground electronic state $\left(S_{0}\right)$ to the origin of the first excited state $\left(S_{1}, v=0 ;{ }^{1} \pi \pi^{*} \leftarrow^{1} \pi \pi\right.$ transition), catechol undergoes $\mathrm{O}-\mathrm{H}$ bond fission mediated via a tunneling mechanism. This occurs beneath an $S_{1} / S_{2}$ conical intersection, where the $S_{2}$ state is a ${ }^{1} \pi \sigma^{*}$ state that is dissociative along the $\mathrm{O}-\mathrm{H}$ coordinate. Importantly, with respect to this present study, our group showed that $\mathrm{O}-\mathrm{H}$ fission occurred on a picosecond (ps) time scale [15], orders of magnitude faster than that seen for other dihydroxybenzenes [17-19]. The object of the present work is to study, in detail, the vibrational motions that occur prior to, and during, the tunneling process.

In order to probe these atomic motions in real time, we utilize the difference in geometry between the excited electronic state $\left(S_{1}\right)$ and the ground cationic state $\left(D_{0}\right)$ as an effective Franck-Condon (FC) detection window.
Following UV irradiation, a coherent superposition of low-lying FC-active vibrational motions is created in $S_{1}$, resulting in the formation of a localized vibrational wave packet. The temporal evolution of the prepared superposition can then be probed by virtue of the nonplanar $\rightarrow$ planar $\left(\mathrm{S}_{1 \text { min }} \rightarrow\right.$ catechol $\left.^{+}\right)$geometry change upon photoionization [Fig. 1(b)]. The evolution of the prepared wave packet allows us to gain important insight into the nuclear motions responsible for energy flow on $S_{1}$ at the very early stages of photodissociation in catechol. We utilize a combination of time-resolved ion-yield (TR-IY) and time-resolved velocity map ion imaging to directly probe, for the first time, these nuclear motions, with complementary theoretical calculations to illuminate the observed dynamics.

The experimental procedure has been described in detail elsewhere $[29,30]$ and thus is only briefly summarized here. Catechol is first seeded into helium ( $\sim 2$ bar) and expanded

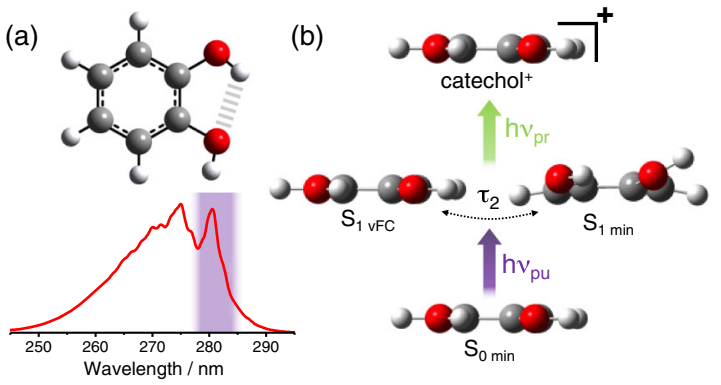

FIG. 1 (color online). (a) Calculated structure of catechol in the $S_{0}$ state accompanied by the vapor phase UV-vis absorption spectrum for catechol (red line). The highlighted region indicates the excitation region for the current study. (b) Schematic representation of the pump-probe process showing the associated nuclear geometries calculated by using the CAM-B3LYP functional with an aug-cc-pVDZ basis set (see Supplemental Material for further details [20]). 


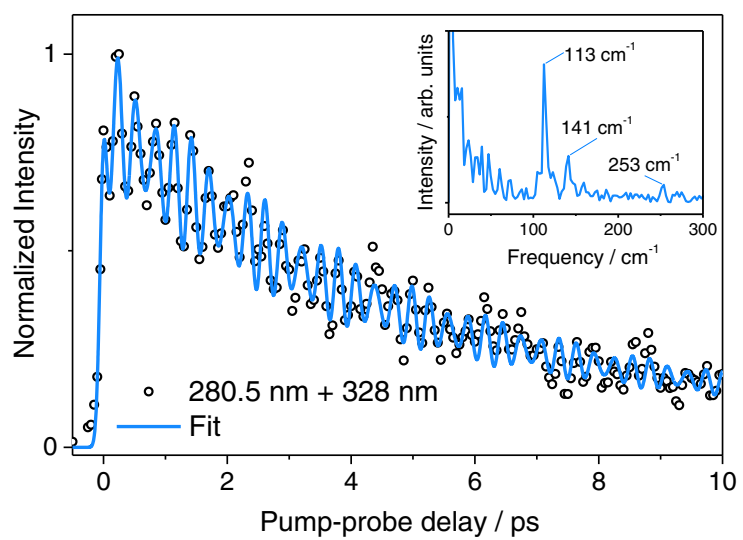

FIG. 2 (color online). TR-IY transient collected from catechol following UV excitation at $280.5 \mathrm{~nm}$ and subsequent photoionization (probing) using $328 \mathrm{~nm}$. A $50 \mathrm{fs}$ step size was used (as indeed elsewhere) to resolve the oscillations. The solid blue line indicates the fit as described in the main text and Ref. [20]. Inset: The FFT of the transient.

into vacuum ( $\sim 10^{-7}$ mbar) by using an Even-Lavie pulsed valve [31]. Following excitation of catechol using a broadband $\left(\sim 500 \mathrm{~cm}^{-1}\right)$ femtosecond (fs) pump pulse $\left(h \nu_{\mathrm{pu}}\right)$, a coherent superposition of low-frequency FC-active modes is prepared on the $S_{1}$ state, creating a vibrational wave packet. The excited wave packet is allowed to evolve over time and is then projected (photoionized) onto the $D_{0}$ state by using a second, time-delayed $(\Delta t)$ fs probe pulse $\left(h \nu_{\mathrm{pr}}\right)$. The resulting catechol ${ }^{+}$ion signal is recorded as a function of pump-probe delay by using a microchannel plate detector coupled to an oscilloscope. The pump pulse is centered around the strongly absorbing $S_{1}$ origin band [Fig. 1(a)], while the probe pulse is selected such that the total energy $E_{\text {tot }}$ is sufficient to photoionize just above the adiabatic ionization potential $\left(\mathrm{IP}_{\mathrm{ad}}\right)$, which in catechol lies at $8.17 \mathrm{eV}$ [32].

To garner further insight into the excited state dynamics, we have also performed complementary calculations using the GAUSSIAN 09 [24] and MOLPRO 2012.1 [25] computational packages in order to generate optimized ground, excited, and ionic state geometries and potential energy cuts (PECs) along the $\mathrm{O}-\mathrm{H}$ coordinate for the ground and excited states. All geometries for catechol were calculated by using GAUSSIAN 09 with the CAM-B3LYP [26] level of theory and an aug-cc-pVDZ basis set. The $S_{1}$ state was also calculated with the M052X [27] and multiconfigurational complete active space self-consistent field (CASSCF) [28] levels of theory [20]. Using the ground state geometry, unrelaxed (rigid body) PECs along the $\mathrm{O}-\mathrm{H}$ stretch were then calculated in MOLPRO 2012.1, by using a combination of the CASSCF method and its second-order perturbation theory extension (CASPT2) using a $(12,10)$ active space [33].

Figure 2 shows a typical TR-IY transient (open circles) recorded following excitation of catechol with $280.5 \mathrm{~nm}$ and probing with $328 \mathrm{~nm}$, such that $E_{\text {tot }}$ is only $0.03 \mathrm{eV}$ above the $\mathrm{IP}_{\mathrm{ad}}$. The overall shape of the transient is as previously reported following excitation at $280.5 \mathrm{~nm}$ and probing with $243 \mathrm{~nm}$; a very fast rise around $\Delta t=0$, the point of temporal overlap between pump and probe beams, which ultimately decays over a period of several ps [15]. However, unlike the previously recorded transient, in which $E_{\text {tot }}$ far exceeded $\mathrm{IP}_{\text {ad }}$ (by $1.35 \mathrm{eV}$ ), immediately apparent now is a superimposed oscillation that appears in the transient. The observed quantum beat pattern is attributed, as noted above, to the variation in the ionization cross section as the excited state wave packet evolves on the $S_{1}$ surface.

By taking the fast Fourier transform (FFT) of the transient data, one can extract the frequencies of any oscillatory components in a given TR-IY transient. In all cases, zero padding of the TR-IY data was used to ensure an appropriate number of data points without artificially reducing the frequency resolution. The FFT for the transient (Fig. 2) is shown in the inset in the same figure and yields two frequencies with associated energies centered at 113 and $141 \mathrm{~cm}^{-1}$ (and with associated periodicities of 295 and $237 \mathrm{fs}$, respectively). Comparison of these frequencies with frequency-resolved spectra of the catechol $S_{1}$ state provides insight into which eigenstates are prepared within the superposition. The observed frequencies are in excellent accord with the band separations in the vibrational progression of the low-lying $\mathrm{O}-\mathrm{H}$ torsional mode $\left(\tau_{2}\right)$, primarily of the free [i.e., nonintramolecular hydrogen bonded, Fig. 1(a)] O-H moiety. Specifically, these frequencies correspond to wave number separations between (i) the $S_{1}$ origin and one quantum of excitation in $\tau_{2}\left(S_{1}, v=0\right.$, and $\tau_{20}{ }^{1+}$, respectively, $\Delta E=115 \mathrm{~cm}^{-1}$ ) and (ii) one and two quanta of excitation in $\tau_{2}\left(\tau_{20}{ }^{1+}\right.$ and $\tau_{20}{ }^{2+}$, respectively, $\Delta E=141 \mathrm{~cm}^{-1}$ ) [16]. Indeed, closer inspection of the FFT also reveals an additional feature, albeit much weaker, with an associated energy around $250 \mathrm{~cm}^{-1}$. This frequency corresponds to the wave number separation between the $S_{1}$ origin and $\tau_{20}{ }^{2+}\left(\Delta E=256 \mathrm{~cm}^{-1}\right)$. The broadness of this peak, and to a lesser extent the peaks at 113 and $141 \mathrm{~cm}^{-1}$, reflects the limited time window over which these beats can be observed, due to the fast overall decay of the $S_{1}$ state.

We are able to model the TR-IY transient by using a decay function convoluted with our instrument response function, which serves to model the overall rise (following the excitation pulse) and decay in the transient, in combination with two cosine functions to model the quantum beats. The cosine functions are themselves superimposed with an exponential decay which serves to dampen the quantum beats (vide infra and [20]). The blue line in Fig. 2 gives the resultant overall fit to the measured transient [34]. The frequencies obtained from the fit align extremely well with those extracted via the FFT, giving values of 113 and $140 \mathrm{~cm}^{-1}$ (periodicities of 295 and $239 \mathrm{fs}$, respectively), adding weight to our assignment of these beats to the progression in the free $\mathrm{O}-\mathrm{H}$ torsional motion. 

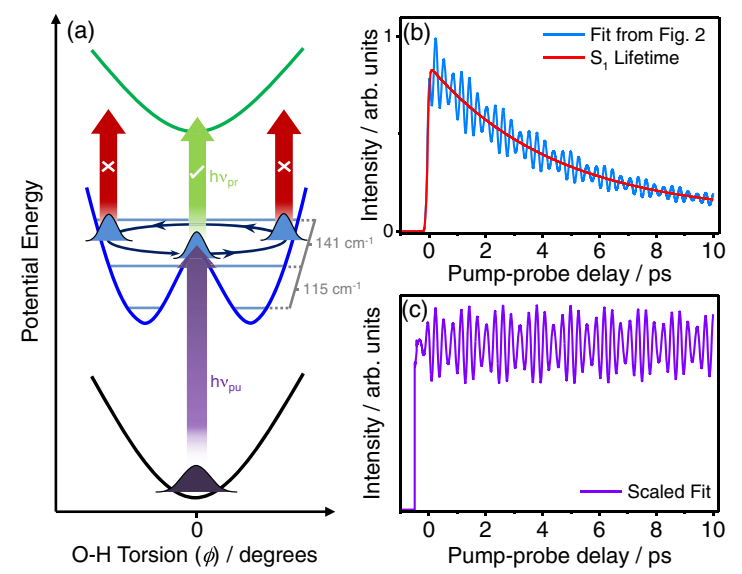

FIG. 3 (color online). (a) Schematic depiction of the excitation mechanism adapted from Refs. [36] and [37]. (b) The fit function from Fig. 2 (blue line) and the same fit but with the oscillatory components removed from the fit (i.e., the $S_{1}$ lifetime; red line). (c) The resulting trace following scaling of the fit from (b); further details in the main text and Ref. [20].

We now consider the origins of these quantum beats, assisted by the schematic shown in Fig. 3(a). Following photoexcitation from the planar $S_{0}$ ground state, a coherent superposition of one and two quanta in the $\mathrm{O}-\mathrm{H}$ torsional mode creates a vibrational wave packet in the nonplanar excited $S_{1}$ state. The final $D_{0}$ state of catechol ${ }^{+}$is planar, and thus the nuclear configuration is once again different, this time between the $S_{1}$ (nonplanar) and $D_{0}$ (planar) states. As such, there is a varying ionization cross section along the torsional coordinate $(\phi)$, which we highlight by the green and red arrows [35]. The localized vibrational wave packet oscillates along $\phi$; however, it can be probed only at or around $\phi=0$, as the probe photon energy is greater than $\mathrm{IP}_{\mathrm{ad}}$; at nonplanar geometries, the probe photon energy is insufficient to overcome $\mathrm{IP}_{\mathrm{ad}}$.

It is possible to attribute the apparent damping in the quantum beats in Fig. 2 to the $S_{1}$ decay or, as previously observed in similar systems, guaiacol and syringol [35], to intramolecular vibrational energy redistribution (IVR), or both. With reference to the frequency-resolved measurements [16,38], within our excitation pulse, the only FC active vibrational modes present are from the short progression in $\tau_{2}$, with only a limited set of these modes within the spectral bandwidth of our excitation pulse $\left(\sim 500 \mathrm{~cm}^{-1}\right)$. This means that any IVR, at first sight, is not possible around the $S_{1}$ origin. Calculations, however, suggest a small number of heavily mixed vibrational modes (reminiscent of those seen in guaiacol, which also has a nonplanar $S_{1}$ state minimum and undergoes Duschinsky mixing [39]), including $\tau_{2}$, that may fall within our excitation window [15,38]. This implies that IVR, while highly restricted, cannot be entirely ruled out.

To explore this further, Fig. 3(b) (blue trace) shows the fit to the transient shown in Fig. 2. If we now scale the overall fit function with respect to the $S_{1}$ decay [Fig. 3(b), red line], this yields the trace shown in Fig. 3(c), comprised solely of the sinusoidal quantum beats, clearly emphasising the persistence of the oscillations for the duration of the excited state lifetime. We can therefore attribute the apparent damping of the quantum beats simply through the fact that the overall $S_{1}$ population decays almost completely within our $10 \mathrm{ps}$ window. The amplitude of the quantum beat oscillations therefore decreases proportionally to the population of $S_{1}$. Predictably, this suggests that there is no IVR, from our excited state population, taking place following photoexcitation to the $S_{1}$ state (vide infra). We note that the $S_{1}$ decay in these, and our previous measurements around the $S_{1}$ origin ( $\left.\sim 8.5 \mathrm{ps}\right)$ [15], accords reasonably well with the excited state lifetimes measured by Weiler et al. [38] following photoexcitation at the $S_{1}$ origin (7 ps), $\tau_{20}{ }^{1+}(11 \mathrm{ps})$, and $\tau_{20}{ }^{2+}(10 \mathrm{ps})$. However, we also note that our broadband pulse excites a superposition of these states at (and around) the $S_{1}$ origin, meaning that our measured $S_{1}$ lifetime is very likely an overall "average" lifetime of these states.

By tuning the excitation pulse wavelength, we are able to manipulate the contribution of one component beat over the other in our coherent superposition. By increasing the excitation wavelength to $281.5 \mathrm{~nm}$ and probing with $326.6 \mathrm{~nm}$ (the total photon energy is once again $8.2 \mathrm{eV}$, $0.03 \mathrm{eV}$ above $\mathrm{IP}_{\mathrm{ad}}$ ), the spectral bandwidth of our excitation pulse no longer encompasses $\tau_{20}{ }^{2+}$ but only $\tau_{20}{ }^{1+}$ and the $S_{1}$ origin. This is manifested as a single quantum beat in the measured transient and is shown in Fig. 4(a) with the inset showing the FFT, which almost exclusively yields a single frequency with an associated energy of $116 \mathrm{~cm}^{-1}$ (i.e., the energy difference between $S_{1}$, $v=0$, and $\tau_{20}{ }^{1+}$, respectively). The solid line is once again a fit to the measured transient, although in this case only a single cosine function was required to model the single quantum beat, damped, once again, by the overall decay in $S_{1}$.

Previous work by King et al. [16] on the photodissociation dynamics of catechol has suggested that the initial torsional excitation can be efficiently redistributed into ring puckering and likely in-plane ring stretching vibrations as the $\mathrm{H}$ atom of the free O-H tunnels beneath the $S_{1} / S_{2}$ conical intersection, en route to $\mathrm{O}-\mathrm{H}$ dissociation. This is evidenced through population of these modes in the catechoxyl radical cofragment. The quantum beats observed here allow us to directly infer the extent of population transfer from the $\mathrm{O}-\mathrm{H}$ torsional motion into any additional modes prior to tunneling. The lack of IVR evidenced in these quantum beats leads us to conclude that the considerable degree of mode mixing already present within the $S_{1}$ manifold $[15,36,38]$ likely also translates into vibrational motion in the catechoxyl radical.

We might anticipate, given the remarkably strong coherence of the initially prepared superposition in catechol, to observe a reflection of this periodicity in any dissociated 

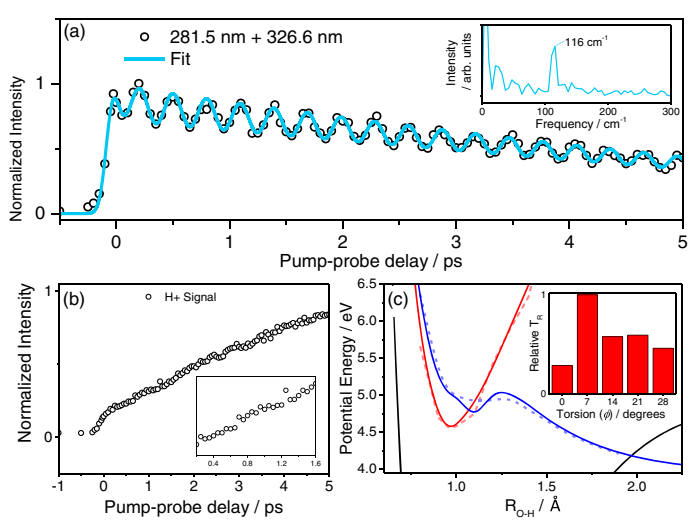

FIG. 4 (color online). (a) The TR-IY transient collected from catechol following excitation and probing with 281.5 and $326.6 \mathrm{~nm}$, respectively. The solid blue line indicates the fit as described in the main text. Inset: The FFT of the transient. (b) Representative $\mathrm{H}^{+}$transient recorded at the same pump wavelength as the TR-IY transient and probing with $243 \mathrm{~nm}$ (to resonantly ionize $\mathrm{H}$ atoms). Inset: Enlarged section of the transient to highlight the lack of stepwise increase in signal. (c) $S_{0}$ (black), $S_{1}$ (red), and $S_{2}$ (blue) PECs along $R_{\mathrm{O}-\mathrm{H}}$ when the molecular geometry is both planar (solid lines) and bent (dashed lines) [20]. Inset: Graph showing the effect $\phi$ has on the tunneling probability $T_{R}$.

$\mathrm{H}$ atoms. A representative $\mathrm{H}^{+}$transient obtained by using time-resolved velocity map ion imaging is shown in Fig. 4(b). It is clear that, within the signal to noise, we are unable to resolve any prevalent stepwise increase in the $\mathrm{H}^{+}$signal. In an attempt to explore this further, we undertook calculations to investigate how the $S_{1}$ state energy landscape changes as a function of the torsional coordinate of the free O-H. Figure 4(c) highlights the $S_{1}$ and $S_{2}$ PECs along $R_{\mathrm{O}-\mathrm{H}}$ when the molecular geometry is both planar $\left(S_{1 \mathrm{vFC}}, \phi \sim 0^{\circ}\right.$; solid lines) and where the free $\mathrm{O}-\mathrm{H}$ lies out of the molecular plane $\left(S_{1 \mathrm{~min}}, \phi \sim 14^{\circ}\right.$; dashed lines). Cursory inspection reveals that, while there is some change to the overall area of the barrier to tunneling, the absolute effect of this change is likely too small for the present measurements to detect. This is highlighted by the graph inset, which shows the relative tunneling probability $\left(T_{R}\right)$, calculated by using a 1D WKB approximation [40], as a function of $\phi$. While $T_{R}$ is lowest at planar geometry, the overall variation in $T_{R}$ is insufficient (certainly within our signal to noise) to be mapped through onto the $\mathrm{H}^{+}$transient. Interestingly, the finding that $T_{R}$ is lowest at planar geometry accords with the measurements of Weiler et al. [38], who find that the lifetime of the $S_{1}$ state is strongly dependent on the degree of planarity; the more planar (i.e., $\phi$ approaches zero), the longer the lifetime measured. We must acknowledge, however, the oversimplicity of these calculations, given that we are effectively freezing all other nuclear coordinates and varying only $\phi$ and $R_{\mathrm{O}-\mathrm{H}}$ of the free $\mathrm{O}-\mathrm{H}$. That being said, the results provide impetus for future calculations incorporating geometry relaxation along all nuclear coordinates.
In conclusion, vibrational wave packets were prepared in the $S_{1}$ state of the catechol chromophore. The subsequent temporal evolution of the superposition shows a strong quantum beat pattern in the parent transient, possessing frequencies in line with the progression in the heavily mixed $\tau_{2}$ mode. The apparent dampening in the quantum beat is attributed to the $S_{1}$ decay, with no evidence of any measurable IVR taking place from the excited state population localized in this mode. Calculations were performed in an effort to characterize the excited state landscape as a function of the torsional angle. We find that, while the tunneling probability $T_{R}$ varies with changing $\phi$, this variance is insufficient to produce resolvable evidence for a stepwise increase in the $\mathrm{H}$ (and thus $\mathrm{H}^{+}$) photofragment.

These results serve to highlight key nuclear motions that are responsible for energy flow in a biological chromophore. They also demonstrate the sensitivity of TR-IY spectroscopy, showing that, given careful choice of experimental parameters, in this case careful selection of probe wavelengths combined with the intrinsic molecular properties, i.e., a change in nuclear geometry between electronic states, it is possible to gain exquisite insight into the concerted atomic motions of a molecule as the wave packet evolves on the excited state surface, while undergoing photodissociation.

We are grateful to Professor Philipp Kukura (Oxford) for helpful discussions. J. D. Y. thanks the University of Warwick for a doctoral training award. M. S. thanks the EPSRC for postdoctoral funding. M. J. P. thanks the ERC for funding under the EU's Seventh Framework Program (FP7/2007-2013)/ERC Grant No. 258990. V. G. S. thanks the EPSRC for an equipment Grant No. (EP/J007153) and the Royal Society for a University Research Fellowship. J. D. Y. and M. S. contributed equally to this work.

*v.stavros@warwick.ac.uk

[1] A. L. Sobolewski, W. Domcke, C. Dedonder-Lardeux, and C. Jouvet, Phys. Chem. Chem. Phys. 4, 1093 (2002).

[2] M. N. R. Ashfold, G. A. King, D. Murdock, M. G. D. Nix, T. A. A. Oliver, and A. G. Sage, Phys. Chem. Chem. Phys. 12, 1218 (2010).

[3] M. N. R. Ashfold, B. Cronin, A. L. Devine, R. N. Dixon, and M. G. D. Nix, Science 312, 1637 (2006).

[4] G. M. Roberts and V. G. Stavros, Chem. Sci. 5, 1698 (2014).

[5] P. Meredith and T. Sarna, Pigment Cell Research 19, 572 (2006).

[6] B. Heggen, Z. Lan, and W. Thiel, Phys. Chem. Chem. Phys. 14, 8137 (2012).

[7] M. K. Shukla and J. Leszczynski, J. Biomol. Struct. Dyn. 25, 93 (2007).

[8] M. Staniforth and V. G. Stavros, Proc. R. Soc. London, Ser. A 469, 20130458 (2013).

[9] C. Su, C. T. Middleton, and B. Kohler, J. Phys. Chem. B 116, 10266 (2012). 
[10] K. de La Harpe and B. Kohler, J. Phys. Chem. Lett. 2, 133 (2011).

[11] A. S. Chatterley, C. W. West, V. G. Stavros, and J. R. R. Verlet, Chem. Sci. 5, 3963 (2014).

[12] A. S. Chatterley, C. W. West, G. M. Roberts, V. G. Stavros, and J. R. R. Verlet, J. Phys. Chem. Lett. 5, 843 (2014).

[13] A. L. Sobolewski and W. Domcke, ChemPhysChem 8, 756 (2007).

[14] A. Huijser, A. Pezzella, and V. Sundstrom, Phys. Chem. Chem. Phys. 13, 9119 (2011).

[15] A. S. Chatterley, J. D. Young, D. Townsend, J. M. Zurek, M. J. Paterson, G. M. Roberts, and V. G. Stavros, Phys. Chem. Chem. Phys. 15, 6879 (2013).

[16] G. A. King, T. A. A. Oliver, R. N. Dixon, and M. N. R. Ashfold, Phys. Chem. Chem. Phys. 14, 3338 (2012).

[17] R. A. Livingstone, J. O.F. Thompson, M. Iljina, R. J. Donaldson, B. J. Sussman, M. J. Paterson, and D. Townsend, J. Chem. Phys. 137, 184304 (2012).

[18] M. Staniforth, A. S. Chatterley, J. D. Young, G. M. Roberts, and V. G. Stavros, Biomed. Spectrosc. Imaging 3, 271 (2014).

[19] J. D. Young, M. Staniforth, A. S. Chatterley, M. J. Paterson, G. M. Roberts, and V. G. Stavros, Phys. Chem. Chem. Phys. 16, 550 (2014).

[20] See Supplemental Material at http://link.aps.org/ supplemental/10.1103/PhysRevLett.114.233001 for calculated minimum energy geometries, details of kinetic fits and information on calculated potential energy surfaces, which includes Refs. [15,21-28].

[21] T. H. Dunning, Jr., J. Chem. Phys. 90, 1007 (1989).

[22] P. Celani and H. J. Werner, J. Chem. Phys. 112, 5546 (2000).

[23] H. J. Werner, Mol. Phys. 89, 645 (1996).

[24] M. J. Frisch et al., Gaussian 09, Revision A.02, Gaussian, Inc., Wallingford CT.

[25] H. J. Werner et al., MOLPRO, version 2012.1, a package of ab initio programs; see http://www.molpro.net.

[26] T. Yanai, D. P. Tew, and N. C. Handy, Chem. Phys. Lett. 393, 51 (2004).
[27] Y. Zhao, N. E. Schultz, and D. G. Truhlar, J. Chem. Theory Comput. 2, 364 (2006)

[28] H. J. Werner and P. J. Knowles, J. Chem. Phys. 82, 5053 (1985)

[29] A. Iqbal, M. S. Y. Cheung, M. G. D. Nix, and V. G. Stavros, J. Phys. Chem. A 113, 8157 (2009).

[30] K. L. Wells, G. Perriam, and V. G. Stavros, J. Chem. Phys. 130, 074308 (2009).

[31] U. Even, J. Jortner, D. Noy, N. Lavie, and C. CossartMagos, J. Chem. Phys. 112, 8068 (2000).

[32] M. Gerhards, S. Schumm, C. Unterberg, and K. Kleinermanns, Chem. Phys. Lett. 294, 65 (1998).

[33] The $(12,10)$ active space consists of the $3 \pi$ bonding orbitals, $3 \pi^{*}$ antibonding orbitals, the $2 \mathrm{n} \pi$ lone pair orbitals on the $\mathrm{O}$ atoms, and the correlated $\sigma$ and $\sigma^{*}$ orbitals associated with the dissociating $\mathrm{O}-\mathrm{H}$ bond (see orbital hypersurfaces in ESI of Ref. [15]).

[34] A similar fitting procedure is outlined in full in the ESI of Ref. [35]. In the case of catechol, we use one fewer exponential decay function, as it appears we are insensitive to the initial geometry rearrangement that is present in syringol and guaiacol.

[35] J. D. Young, M. Staniforth, J. C. Dean, G. M. Roberts, F. Mazzoni, T. N. V. Karsili, M. N. R. Ashfold, T. S. Zwier, and V. G. Stavros, J. Phys. Chem. Lett. 5, 2138 (2014).

[36] T. Bürgi and S. Leutwyler, J. Chem. Phys. 101, 8418 (1994).

[37] M. Gerhards, W. Perl, S. Schumm, U. Henrichs, C. Jacoby, and K. Kleinermanns, J. Chem. Phys. 104, 9362 (1996).

[38] M. Weiler, M. Miyazaki, G. Féraud, S.-i. Ishiuchi, C. Dedonder, C. Jouvet, and M. Fujii, J. Phys. Chem. Lett. 4, 3819 (2013).

[39] J. C. Dean, P. Navotnaya, A. P. Parobek, R. M. Clayton, and T. S. Zwier, J. Chem. Phys. 139, 144313 (2013).

[40] R. J. Le Roy and W. K. Liu, J. Chem. Phys. 69, 3622 (1978). 